\title{
Age and Racial Disparities in Telehealth Use Among People with HIV During the COVID-19 Pandemic
}

\author{
Eleanor E. Friedman ${ }^{1,2}\left(\right.$ D $\cdot$ Samantha A. Devlin ${ }^{1,2} \cdot$ Sarah F. Gilson $^{2,3} \cdot$ Jessica P. Ridgway $^{1,2}$ \\ Accepted: 23 January 2022 / Published online: 8 February 2022 \\ (c) The Author(s), under exclusive licence to Springer Science+Business Media, LLC, part of Springer Nature 2022
}

\begin{abstract}
The COVID-19 pandemic resulted in widespread telehealth expansion. To determine telehealth uptake and potential sociodemographic differences in utilization among people with HIV $(\mathrm{PwH})$, we examined HIV care appointments at the University of Chicago Medicine, an urban tertiary hospital. Visits between March 15th and September 9th for 2019 and 2020 were categorized as in-person, telehealth, and within telehealth, video, and phone. Differences in visit types were modeled using logistic regression to examine associations with demographics, insurance type, and HIV risk transmission category. Telehealth appointments were more likely for those aged 46-60 versus those 31-45 [46-60; AOR 1.89 95\% CI $(1.14,3.15)$ ]. Black race and participants of other races were less likely to use telehealth compared to whites [Black: AOR 0.33 95\% CI $(0.16,0.64)$, other: AOR $0.1095 \%$ CI $(0.02,0.34)]$. Future studies should continue to examine potential disparities in telehealth use among $\mathrm{PwH}$, including age and racial differences.
\end{abstract}

Keywords Telehealth $\cdot$ HIV $\cdot$ Disparities $\cdot$ Infectious disease $\cdot$ COVID-19

\section{Introduction}

The COVID-19 pandemic greatly disrupted healthcare systems worldwide [1]. This disruption extended to ambulatory care visits, which were either canceled or delayed early in the COVID-19 pandemic or converted from in-person appointments to telehealth appointments later in the pandemic $[1,2]$. In this study, telehealth is defined as a long distance medical appointment by telephone, video, or other telecommunication [3].

Both initiation of telehealth and rapid increases in telehealth appointments due to the COVID-19 pandemic have been seen, but disparities exist in patient utilization of telehealth [2, 4-7]. Several studies have found that older patients, non-English speaking patients, and patients with public insurance were less likely to use telehealth as

Eleanor E. Friedman

Efriedman@medicine.bsd.uchicago.edu

Chicago Center for HIV Elimination, Chicago, IL, USA

2 Department of Medicine, University of Chicago, Chicago, IL, USA

3 Center for Healthcare Delivery Science and Innovation, University of Chicago Medicine, Chicago, IL, USA compared to in-person visits $[4,5,7-9]$. These disparities could be due to differences in the patient populations; for instance, older and unstably housed patients and those with low broadband access are less likely to use telehealth, particularly video visits, compared to younger patients and those with higher income $[4,9,10]$.

The switch from in-person to telehealth visits during the pandemic has not been studied equally across all specialties. Few studies have examined HIV care appointments to determine the uptake of telehealth and possible differences among people living with HIV $(\mathrm{PwH})$ who utilized telehealth during the COVID-19 pandemic [11]. PwH may face particular challenges such as transportation issues, stigma, insufficient insurance, and mental illness/substance use disorders that make clinic attendance difficult even in ordinary times [12, 13]. These circumstances, in addition to concerns regarding SARS-CoV-2 infection, may have differentially impacted appointment attendance and type of appointment during the pandemic. Thus, we examined total visits, visit type (inperson vs. telehealth, telephone vs. video), and associations between total visits and visit type with patient sociodemographic factors to describe healthcare usage of $\mathrm{PwH}$ before and during the COVID-19 pandemic at an urban academic medical center. 


\section{Methods}

We examined encounters for $\mathrm{PwH}$ who attended appointments in the Infectious Diseases (ID) department Ryan White adult HIV clinic at the University of Chicago Medicine (UCM), a major provider of HIV care on the south side of Chicago. To accurately compare pre-pandemic and pandemic periods, data were limited to the time between March 15th and September 9th for 2019 and 2020. March 15th, 2020 was chosen as the start date of the pandemic period to reflect the March 18th shelter at home order issued in Chicago [14].

In this study, we refer to care provided during the COVID-19 pandemic in place of in-person appointments as "telehealth." Although the term "telemedicine" refers to video visits specifically, "telehealth" is a broader term that includes using telephone, video, or other telecommunication to facilitate long-distance health care [3].

At our institution, all ambulatory clinics could provide video visits on and after April 6th, 2020. All persons 18 years or older who had an ambulatory visit at UCM and had either a diagnosis code for HIV or HIV identified as a problem in their electronic medical record (EMR) were included in the study. All information was gathered from EMRs. Patient sociodemographic characteristics including age, race, ethnicity, insurance type, and HIV transmission category were examined for associations with visit attendance and visit type. Age was subdivided into the following categories: $18-30$ years, $31-45$ years, 46-60 years, and 61 years or older. Race was categorized into African American/Black, white, and other (including unknown, patient declined, more than one race, Asian/ Mideast Indian, American Indian/Alaskan Native, and Native Hawaiian/Pacific Islander). Insurance was categorized into Medicare (including Medicare-Medicaid Alignment Initiative), Medicaid, commercial, and other. HIV transmission category was categorized into men who have sex with men (MSM), intravenous drug use (IVDU), heterosexual sex, other (including Hemophilia/Coagulation disorder, prenatal, transfusion), and unknown. We included HIV transmission category as a variable in this analysis due to the association between certain transmission categories, such as IVDU, and difficulties in retention in HIV care [15-18]. Visits were classified as in-person or telehealth, with further subdivisions of telehealth into video or telephone. This project underwent formal review and received a determination of Quality Improvement status according to UCM institutional policy. Thus, this initiative was not considered human subjects research and was not reviewed by the Institutional Review Board.

Data analysis included describing the data using frequencies, percentages, and medians with interquartile intervals. Hypothesis tests were conducted using chi squared or Fisher's exact tests and Student's $t$ tests. We used logistic regression models to measure bivariate associations using odds ratios (OR) and $95 \%$ confidence intervals $(95 \% \mathrm{CI})$. Variables that were significant at the bivariate level were added to a multivariate logistic regression model, producing adjusted odds ratios (AOR) and 95\% CI. If significant differences between unadjusted and adjusted models were not seen, adjusted results were presented. All data analysis was completed in RStudio version 3.6.3.

\section{Results}

Healthcare utilization, as measured both by the number of unique patients and the number of total appointments among $\mathrm{PwH}$, decreased in the pandemic period from the prepandemic period. In the pre-pandemic period 468 patients attended 740 total appointments, whereas during the pandemic period 388 patients attended 520 total appointments (including both in-person and telehealth visits), a drop of $17.1 \%$ and $29.7 \%$ respectively.

In terms of patient retention between periods, out of 594 total patients, 206 patients only attended appointments in the pre-pandemic period (34.7\%), 126 only attended during the pandemic period $(21.2 \%)$, and $44.1 \%$ of patients who attended in the pre-pandemic period also attended during the pandemic period. There were few significant differences among patient demographics between periods, although the number of appointments for persons aged 46-60 decreased in 2020 as compared to 2019 [AOR $0.7195 \%$ CI $(0.52$, 0.97)], and we saw fewer persons whose HIV transmission category was other and more whose transmission category was unknown in 2020 [other: AOR $0.4495 \% \mathrm{CI}(0.23,0.82)$ ] unknown [AOR 4.84 95\% CI $(1.41,22.24)$ ] (Table 1).

In the pre-pandemic period, only in-person appointments were available in the ID clinic. During the pandemic period, visit type was nearly equally divided between in-person (291, $56.0 \%)$ and telehealth appointments $(229,44.0 \%)$. Some patients utilized only in-person appointments (190 patients, $49.0 \%$ ) or only telehealth (170 patients, $43.8 \%$ ) while a minority utilized both (28 patients, $7.2 \%$ ). When comparing telehealth appointments to in-person appointments during the pandemic period, age-related associations were seen. Telehealth appointments were significantly more likely among patients aged 46-60 compared to those aged 31-45 in both unadjusted and adjusted analyses [AOR 1.89 95\% CI $(1.14,3.15)]$. In unadjusted results, appointments among persons 61 years and older were also significantly more likely to be virtual when compared to those aged 31-45 [OR 2.09 95\% CI $(1.23,3.58)]$, although this association did not remain significant in the multivariate model [AOR $1.6595 \% \mathrm{CI}(0.88,3.10)]$. There were also racial disparities related to telehealth usage, with Black 
Table 1 Total infectious disease appointments for $\mathrm{PwH}$ during the pre-pandemic $(3 / 15 / 2019$ 9/9/2020) and pandemic $(3 / 15 / 2020-9 / 9 / 2021)$ study periods $(\mathrm{n}=1260)$

\begin{tabular}{|c|c|c|c|c|}
\hline & $\begin{array}{l}2019 \text { total } \\
\text { appointments } \\
(\mathrm{n}=740)\end{array}$ & $\begin{array}{l}2020 \text { total } \\
\text { appointments } \\
(\mathrm{n}=520)\end{array}$ & $\begin{array}{l}\text { Adjusted odds ratios } 2019 \\
\text { vs } 2020 \text { AOR }(95 \% \mathrm{CI})\end{array}$ & $\begin{array}{l}\text { Wald p-values } \\
\text { (Adjusted } \\
\text { model)* }\end{array}$ \\
\hline \multicolumn{5}{|l|}{ Age } \\
\hline $18-30$ & $161(21.8 \%)$ & $136(26.2 \%)$ & $1.07(0.76,1.50)$ & 0.70 \\
\hline $31-45$ & $167(22.6 \%)$ & $137(26.4 \%)$ & Referent & Referent \\
\hline $46-60$ & $265(35.8 \%)$ & $153(29.4 \%)$ & $0.71(0.52,0.97)$ & 0.03 \\
\hline $61+$ & $147(19.9 \%)$ & $94(18.1 \%)$ & $0.85(0.57,1.25)$ & 0.41 \\
\hline \multicolumn{5}{|l|}{ Sex } \\
\hline Male & $473(63.9 \%)$ & $346(66.5 \%)$ & $0.99(0.70,1.39)$ & 0.95 \\
\hline Female & $267(36.1 \%)$ & $174(33.5 \%)$ & Referent & Referent \\
\hline \multicolumn{5}{|l|}{ Race } \\
\hline White & $83(11.2 \%)$ & $53(10.2 \%)$ & Referent & Referent \\
\hline Black & $628(84.9 \%)$ & $445(85.6 \%)$ & $1.13(0.76,1.68)$ & 0.56 \\
\hline Other & $29(3.9 \%)$ & $22(4.2 \%)$ & $1.18(0.60,2.34)$ & 0.63 \\
\hline \multicolumn{5}{|l|}{ Insurance } \\
\hline Commercial & $197(26.6 \%)$ & $153(29.4 \%)$ & Referent & Referent \\
\hline Medicaid & $292(39.5 \%)$ & $210(40.4 \%)$ & $0.89(0.65,1.17)$ & 0.44 \\
\hline Medicare & $244(33.0 \%)$ & $149(28.7 \%)$ & $0.89(0.61,1.18)$ & 0.36 \\
\hline Other & $7(0.9 \%)$ & $8(1.5 \%)$ & $1.31(0.45,3.85)$ & 0.62 \\
\hline \multicolumn{5}{|c|}{ Transmission category } \\
\hline MSM & $338(45.7 \%)$ & $254(48.8 \%)$ & $1.00(0.72,1.41)$ & 0.98 \\
\hline IVDU & $46(6.2 \%)$ & $21(4.0 \%)$ & $0.71(0.39,1.25)$ & 0.24 \\
\hline Heterosexual sex & $311(42.0 \%)$ & $220(42.3 \%)$ & Referent & Referent \\
\hline Other & $42(5.7 \%)$ & $15(2.9 \%)$ & $0.44(0.23,0.82)$ & 0.01 \\
\hline Unknown & $3(0.4 \%)$ & $10(1.9 \%)$ & $4.84(1.41,22.24)$ & 0.02 \\
\hline
\end{tabular}

*Significant p-values are those $\leq 0.05$ patients and patients categorized as other race less likely to attend virtual appointments than white patients. These findings were seen in both unadjusted and adjusted results [Black: AOR 0.33 95\% CI $(0.16,0.64)$, other: AOR $0.1095 \%$ CI $(0.02$, $0.34)]$. No differences between in-person and telehealth visits were seen with regard to insurance coverage or HIV transmission category (Table 2).

When visits during the pandemic were restricted to just telehealth, similar age-related associations were also seen. Patients aged 46-60 and those 61 years and older were significantly less likely to attend a video visit versus a telephone visit when compared to those aged 31-45 [46-60; AOR 0.43 95\% CI $(0.18,0.97), 61$ and older; AOR $0.1995 \%$ CI $(0.07,0.50)]$. No significant differences were seen between telephone and video visits for characteristics such as race, insurance category, or HIV transmission category (Table 2).

\section{Discussion}

In our examination of telehealth patterns and disparities among PwH during the COVID-19 pandemic, the number of PwH who attended in-person appointments was almost the same as those who were seen via telehealth. This ratio is similar to the mix of in-person and telehealth usage reported by the Kaiser Family Foundation in their survey of Ryan White providers [19]. Although other HIV care providers have expressed concern regarding possible disparities in telehealth usage among PwH or have measured their acceptability of telehealth, our study aimed to confirm and quantify these disparities [3, 20-23].

In our study, we found that older persons were more likely to attend virtual visits compared to younger patients, unlike some studies of non-HIV populations $[4,7,10]$. However, other studies also found that older persons were more likely to use telehealth than younger people [2, 8]. Additionally, we found that the preference for telehealth among older patients was driven by telephone rather than video visits, which has been seen in studies of the general population as well [4, $6,8,24]$. Older patients' preference of telehealth over inperson visits could have resulted from early and persistent news coverage highlighting the risk of severe COVID-19 disease among older persons with comorbidities, as well as the unclear risk of contracting SARS-CoV-2 infection for $\mathrm{PwH}$ [25]. The preference of these same patients for telephone rather than video telehealth visits could be due 
Table 2 Infectious disease appointments among PwH during the pandemic period examining type of appointment $(\mathrm{n}=520)$

\begin{tabular}{|c|c|c|c|c|c|c|c|c|}
\hline & $\begin{array}{l}2020 \text { in } \\
\text { person } \\
\text { appointments } \\
(\mathrm{n}=291 \text {, } \\
56.0 \%)\end{array}$ & $\begin{array}{l}2020 \text { virtual } \\
\text { appointments } \\
(\mathrm{n}=229 \\
44.0 \%)\end{array}$ & $\begin{array}{l}\text { Adjusted odds } \\
\text { ratios virtual } \\
\text { vs in person } \\
\text { AOR }(95 \% \mathrm{CI})\end{array}$ & $\begin{array}{l}\text { Wald p-values } \\
\text { (Adjusted } \\
\text { model)* }\end{array}$ & $\begin{array}{l}2020 \text { phone } \\
\text { appoint- } \\
\text { ments }(\mathrm{n}=91 \text {, } \\
39.7 \%)\end{array}$ & $\begin{array}{l}2020 \text { video } \\
\text { appointments } \\
(\mathrm{n}=138, \\
60.3 \%)\end{array}$ & $\begin{array}{l}\text { Adjusted odds } \\
\text { ratios video vs } \\
\text { phone AOR } \\
(95 \% \mathrm{CI})\end{array}$ & $\begin{array}{l}\text { Wald p-values } \\
\text { (Adjusted } \\
\text { model)* }\end{array}$ \\
\hline \multicolumn{9}{|l|}{ Age } \\
\hline $18-30$ & $95(32.7 \%)$ & $41(17.9 \%)$ & $\begin{array}{l}0.85(0.50 \\
1.44)\end{array}$ & 0.55 & $18(19.8 \%)$ & $23(16.7 \%)$ & $\begin{array}{l}0.55(0.20 \\
1.45)\end{array}$ & 0.23 \\
\hline $31-45$ & $86(29.6 \%)$ & $51(22.3 \%)$ & Referent & Referent & $12(13.2 \%)$ & $39(28.3 \%)$ & Referent & Referent \\
\hline $46-60$ & $68(23.4 \%)$ & $85(37.1 \%)$ & $\begin{array}{l}1.89(1.14, \\
3.15)\end{array}$ & 0.01 & $33(36.3 \%)$ & $52(37.7 \%)$ & $\begin{array}{l}0.43(0.18 \\
0.97)\end{array}$ & 0.05 \\
\hline $61+$ & $42(14.4 \%)$ & $52(22.7 \%)$ & $\begin{array}{l}1.65(0.88, \\
3.10)\end{array}$ & 0.12 & $28(30.8 \%)$ & $24(17.4 \%)$ & $\begin{array}{l}0.19(0.07 \\
0.5)\end{array}$ & 0.001 \\
\hline \multicolumn{9}{|l|}{ Sex } \\
\hline Male & $194(66.7 \%)$ & $152(66.4 \%)$ & $\begin{array}{l}1.18(0.68 \\
2.07)\end{array}$ & 0.56 & $35(38.5 \%)$ & $42(30.4 \%)$ & $\begin{array}{l}1.52(0.66 \\
3.58)\end{array}$ & 0.33 \\
\hline Female & $97(33.3 \%)$ & $77(33.6 \%)$ & Referent & Referent & $56(61.5 \%)$ & $96(69.6 \%)$ & Referent & Referent \\
\hline \multicolumn{9}{|l|}{ Race } \\
\hline White & $15(5.2 \%)$ & $38(16.6 \%)$ & Referent & Referent & $11(12.1 \%)$ & $27(19.6 \%)$ & Referent & Referent \\
\hline Black & $258(88.7 \%)$ & $187(81.7 \%)$ & $\begin{array}{l}0.33(0.16 \\
0.64)\end{array}$ & 0.002 & $77(84.6 \%)$ & $110(79.7 \%)$ & $\begin{array}{l}0.55(0.21 \\
1.34)\end{array}$ & 0.20 \\
\hline Other & $18(6.2 \%)$ & $4(1.8 \%)$ & $\begin{array}{l}0.10(0.02 \\
0.34)\end{array}$ & 0.001 & $3(3.3 \%)$ & $1(0.7 \%)$ & $\begin{array}{l}0.14(0.01, \\
1.34)\end{array}$ & 0.12 \\
\hline \multicolumn{9}{|l|}{ Insurance } \\
\hline Commercial & $81(27.8 \%)$ & $72(31.4 \%)$ & Referent & Referent & $24(26.4 \%)$ & $48(34.8 \%)$ & Referent & Referent \\
\hline Medicaid & $140(48.1 \%)$ & $70(30.6 \%)$ & $\begin{array}{c}0.76(0.48 \\
1.22)\end{array}$ & 0.26 & $32(35.2 \%)$ & $38(27.5 \%)$ & $\begin{array}{l}0.57(0.26 \\
1.24)\end{array}$ & 0.16 \\
\hline Medicare & $65(22.3 \%)$ & $84(36.7 \%)$ & $\begin{array}{l}1.35(0.80 \\
2.28)\end{array}$ & 0.26 & $32(35.2 \%)$ & $52(37.7 \%)$ & $\begin{array}{l}1.34(0.63, \\
2.87)\end{array}$ & 0.45 \\
\hline Other & $5(1.7 \%)$ & $3(1.3 \%)$ & $\begin{array}{c}0.99(0.19 \\
4.35)\end{array}$ & 0.99 & $3(3.3 \%)$ & $0(0.0 \%)$ & NA & NA \\
\hline \multicolumn{9}{|c|}{ Transmission category } \\
\hline MSM & $146(50.2 \%)$ & $108(47.2 \%)$ & $\begin{array}{c}0.77(0.44 \\
1.35)\end{array}$ & 0.36 & $39(42.9 \%)$ & $69(50.0 \%)$ & $\begin{array}{c}0.80(0.33 \\
1.86 .)\end{array}$ & 0.98 \\
\hline IVDU & $14(4.8 \%)$ & $7(3.1 \%)$ & $\begin{array}{l}0.40(0.14 \\
1.08)\end{array}$ & 0.08 & $4(4.4 \%)$ & $3(2.2 \%)$ & $\begin{array}{l}0.66(0.11, \\
3.48)\end{array}$ & 0.62 \\
\hline $\begin{array}{l}\text { Heterosexual } \\
\text { sex }\end{array}$ & $118(40.5 \%)$ & $102(44.5 \%)$ & Referent & Referent & $44(48.4 \%)$ & $56(40.6 \%)$ & Referent & Referent \\
\hline Other & $8(2.7 \%)$ & $7(3.1 \%)$ & $\begin{array}{c}2.15(0.69 \\
6.69)\end{array}$ & 0.18 & $2(2.2 \%)$ & $5(3.6 \%)$ & $\begin{array}{c}2.09(0.37 \\
16.71)\end{array}$ & 0.43 \\
\hline Unknown & $5(1.7 \%)$ & $5(2.2 \%)$ & $\begin{array}{l}0.90(0.22, \\
3.66)\end{array}$ & 0.88 & $2(2.2 \%)$ & $5(3.6 \%)$ & $\begin{array}{l}0.77(0.11, \\
6.65)\end{array}$ & 0.79 \\
\hline
\end{tabular}

*Significant p-values are those $\leq 0.05$

to discomfort with the newer technology of video calls as opposed to the more traditional audio phone calls [26, 27]. In our study, the age group that most preferred video visits was the one that contained patients aged $31-45$ years.

We also observed racial disparities in terms of visit type, with Black patients and patients classified as other race being less likely to use telehealth compared to white patients. Our hospital serves a majority Black population from the south side of Chicago, an area with economic challenges that could have impacted appointment attendance and type. Lack of internet may have prevented telehealth usage, while use of public transit to attend inperson appointments, as well as fears of increased SARSCoV-2 risk associated with the hospital setting may have resulted in overall lack of healthcare utilization among PwH during the pandemic. It is also possible that $\mathrm{PwH}$ acquiring SARS-CoV-2 was a factor that drove telehealth use among PwH. We have previously examined patterns in SARS-CoV-2 testing among $\mathrm{PwH}$ at our institution during this time period and found that overall SARS-CoV-2 
positivity among $\mathrm{PwH}$ was $7.2 \%$ [28]. Most of these patients were Black (90.3\%) and had a median age of 49 (34-59) years of age, suggesting to us that it is unlikely that infection with SARS CoV-2 is responsible for the racial disparity in telehealth use that we observed. Unfortunately, SARS CoV-2 test result data were not available in the dataset used for the current study, so we cannot confirm this finding.

Other studies have also found racial associations with appointment type in non-HIV populations, with white patients representing a higher proportion of telemedicine visits than other racial/ethnic groups [9] and Black race being associated with nearly twice the odds of completing a telephone visit versus a video visit when compared to whites [6]. For COVID-19 care in particular, fewer Black patients accessed telemedicine compared to white patients, even though Black and Latinx populations were at greater risk for COVID-19 morbidity and mortality [29]. These racial and ethnic differences in telehealth use are likely due to structural factors including smart phone ownership (which is lower among Black patients) and lack of broadband access [30-32]. It may also be due to factors such as inability to obtain privacy at home or at work for a telehealth visit or cultural preference for in-person appointments. These financial and technological barriers can be addressed by interventions at the federal, state and local level, as well as by promoting and incorporating feedback regarding telehealth services among disadvantaged minorities [33].

Other than age and racial differences, we did not find any additional disparities among telehealth usage. These findings are reassuring, as additional telehealth barriers, such as technical issues, low technological literacy, lack of human connection, and concerns regarding privacy on sexual health matters could have presented problems for $\mathrm{PwH}[3$, 20]. Moreover, it is also reassuring that we did not observe a change in insurance coverage between the pre-pandemic and pandemic periods, as has been reported by some HIV care sites [19]. Other studies have found that patients with public insurance were less likely to use telemedicine [5, 7] and were more likely to have telephone encounters rather than video encounters $[6,8,24]$. However, our findings suggest that Medicaid and Medicare coverage for telehealth successfully facilitated continuity of HIV care during the pandemic, which is critical given the high rate of Medicaid insurance utilization in our clinic [34]. We did observe some differences in appointments between the pre-pandemic and pandemic periods, including a decrease in appointments for patients aged 46-60 years in 2020, as well as reduced appointments among those whose HIV transmission category was other and an increase for those whose transmission category was unknown. It is possible that these effects were due to low sample size, especially for those whose risk was unknown, and should be examined further in larger
HIV positive cohorts. In fact, we saw few demographic differences between study periods other than the decrease in appointments for patients aged 46-60 years in 2020.

Our study was conducted over a longer time period (6 months) than other studies examining disparities in telehealth usage among the general population during the COVID-19 pandemic, which may have lessened the impact of the initial disparities seen at the sudden onset of the pandemic. However, our study highlights disparities that still remain after the initial implementation period of telehealth expansion at a large healthcare system. Furthermore, early in the pandemic, it was unclear whether $\mathrm{PwH}$ were uniquely vulnerable to SARS-CoV-2 infection, which may explain the decrease in appointment attendance, as well as appointment type.

Future studies should continue to examine disparities in telehealth usage, specifically among PwH. Most experts recommend keeping telehealth as a permanent option for HIV care [3] and including it when measuring retention in care [21]. Although our study found patients did not often utilize both in-person and telehealth visits, this observation likely resulted from suspension of normal laboratory testing of PwH during the pandemic [22]. Telehealth in non-pandemic times may be more interspersed with in-person visits to monitor CD4 T cell counts and HIV viral load levels.

\section{Conclusions}

In an examination of total visit volume and visit type among PwH during time periods before and during the COVID-19 pandemic, we observed age and racial differences in terms of in-person versus telehealth appointments. We are encouraged by the lack of disparity in telehealth usage with regard to insurance coverage, as telehealth may remain a staple in HIV care after the pandemic. As such, insurance plans, especially Medicaid and Medicare, should continue to include it in their covered services. These findings demonstrate that healthcare systems should include varied visit options (telephone and video), as well as offer in-person appointments for PwH when safe to do so.

Funding Funding sources for the authors include the following, Gilead Sciences Focus Project funding, Grant No. 1K23MH121190-01.

\section{Declarations}

Conflict of interest The authors attest no direct conflicts of interests.

Research Involving Human and Animal Participants This project underwent formal review and received a determination of Quality Improvement status according to UCM institutional policy. Thus, this initiative 
was not considered human subjects research and was not reviewed by the Institutional Review Board.

\section{References}

1. Provenzano DA, Sitzman BT, Florentino SA, Buterbaugh GA. Clinical and economic strategies in outpatient medical care during the COVID-19 pandemic. Reg Anesth Pain Med. 2020;45(8):579-85.

2. Gilson SF, Umscheid CA, Laiteerapong N, Ossey G, Nunes KJ, Shah SD. Growth of ambulatory virtual visits and differential use by patient sociodemographics at one urban academic medical center during the COVID-19 pandemic: retrospective analysis. JMIR Med Inform. 2020;8(12):e24544.

3. Budak JZ, Scott JD, Dhanireddy S, Wood BR. The impact of COVID-19 on HIV care provided via telemedicine-past, present, and future. Curr HIV/AIDS Rep. 2021;18(2):98-104.

4. Rodriguez JA, Betancourt JR, Sequist TD, Ganguli I. Differences in the use of telephone and video telemedicine visits during the COVID-19 pandemic. Am J Manag Care. 2021;27(1):21-6.

5. Tilden DR, Datye KA, Moore DJ, French B, Jaser SS. The rapid transition to telemedicine and its effect on access to care for patients with type 1 diabetes during the COVID-19 pandemic. Diabetes Care. 2021. https://doi.org/10.2337/dc20-2712.

6. Wegermann K, Wilder JM, Parish A, et al. Racial and socioeconomic disparities in utilization of telehealth in patients with liver disease during COVID-19. Dig Dis Sci. 2021. https://doi.org/10. 1007/s10620-021-06842-5.

7. Haynes SC, Kompala T, Neinstein A, Rosenthal J, Crossen S. Disparities in telemedicine use for subspecialty diabetes care during COVID-19 shelter-in-place orders. J Diabetes Sci Technol. 2021. https://doi.org/10.1177/1932296821997851.

8. Pierce RP, Stevermer JJ. Disparities in use of telehealth at the onset of the COVID-19 public health emergency. J Telemed Telecare. 2020. https://doi.org/10.1177/1357633X20963893.

9. Nouri S, Khoong E, Lyles C, Karliner L. Addressing equity in telemedicine for chronic disease management during the Covid-19 pandemic. NEJM Catal Innov Care Deliv. 2020;4(1):3.

10. Ferguson JM, Jacobs J, Yefimova M, Greene L, Heyworth L, Zulman DM. Virtual care expansion in the Veterans Health Administration during the COVID-19 pandemic: clinical services and patient characteristics associated with utilization. J Am Med Inform Assoc. 2021;28(3):453-62.

11. Ennis N, Armas L, Butame S, Joshi H. Factors Impacting video telehealth appointment completion during COVID-19 pandemic among people living with HIV in a community-based health system. AIDS Behav. 2021. https://doi.org/10.1007/s10461-021-03394-7.

12. Yehia BR, Stewart L, Momplaisir F, et al. Barriers and facilitators to patient retention in HIV care. BMC Infect Dis. 2015;15:246.

13. Dombrowski JC, Simoni JM, Katz DA, Golden MR. Barriers to HIV care and treatment among participants in a public health HIV care relinkage program. AIDS Patient Care STDS. 2015;29(5):279-87.

14. Chicago Co. Public Health Order No. 2020-1: Shelter in place for COVID-19 illness. In:2020.

15. Bulsara SM, Wainberg ML, Newton-John TRO. Predictors of adult retention in HIV care: a systematic review. AIDS Behav. 2018;22(3):752-64.

16. Nosyk B, Lourenço L, Min JE, Shopin D, Lima VD, Montaner JS. Characterizing retention in HAART as a recurrent event process: insights into 'cascade churn.' AIDS. 2015;29(13):1681-9.

17. Rebeiro P, Althoff KN, Buchacz K, et al. Retention among North American HIV-infected persons in clinical care, 2000-2008. J Acquir Immune Defic Syndr. 2013;62(3):356-62.
18. Adams JW, Brady KA, Michael YL, Yehia BR, Momplaisir FM. Postpartum engagement in HIV care: an important predictor of long-term retention in care and viral suppression. Clin Infect Dis. 2015;61(12):1880-7.

19. Dawson L, Kates J. Delivering HIV Care and Prevention in the COVID Era: A National Survey of Ryan White Providers. 2020

20. Auchus IC, Jaradeh K, Tang A, Marzan J, Boslett B. Transitioning to telehealth during the COVID-19 pandemic: patient perspectives and attendance at an HIV clinic in San Francisco. AIDS Patient Care STDS. 2021;35(7):249-54.

21. Dandachi D, Freytag J, Giordano TP, Dang BN. It is time to include telehealth in our measure of patient retention in HIV care. AIDS Behav. 2020;24(9):2463-5.

22. Rogers BG, Coats CS, Adams E, et al. Development of telemedicine infrastructure at an LGBTQ+ clinic to support HIV prevention and care in response to COVID-19, providence, RI. AIDS Behav. 2020;24(10):2743-7.

23. Dandachi D, Dang BN, Lucari B, Teti M, Giordano TP. Exploring the attitude of patients with HIV about using telehealth for HIV care. AIDS Patient Care STDS. 2020;34(4):166-72.

24. Gursky JM, Boro A, Escalante S, et al. Disparities in access to neurologic telemedicine during the COVID-19 pandemic: a Bronx tale. Neurol Clin Pract. 2021;11(2):e97-101.

25. Anderson KE, McGinty EE, Presskreischer R, Barry CL. Reports of forgone medical care among US adults during the initial phase of the COVID-19 pandemic. JAMA Netw Open. 2021;4(1):e2034882.

26. Fischer SH, Ray KN, Mehrotra A, Bloom EL, Uscher-Pines L. Prevalence and characteristics of telehealth utilization in the United States. JAMA Netw Open. 2020;3(10):e2022302.

27. Khoong EC, Butler BA, Mesina O, et al. Patient interest in and barriers to telemedicine video visits in a multilingual urban safety-net system. J Am Med Inform Assoc. 2021;28(2):349-53.

28. Friedman EE, Devlin SA, McNulty MC, Ridgway JP. SARS-CoV-2 percent positivity and risk factors among people with HIV at an urban academic medical center. PLoS ONE. 2021;16(7):e0254994.

29. Chunara R, Zhao Y, Chen J, et al. Telemedicine and healthcare disparities: a cohort study in a large healthcare system in New York City during COVID-19. J Am Med Inform Assoc. 2021;28(1):33-41.

30. Greenberg-Worisek AJ, Kurani S, Finney Rutten LJ, Blake KD, Moser RP, Hesse BW. Tracking healthy people 2020 internet, broadband, and mobile device access goals: an update using data from the health information national trends survey. J Med Internet Res. 2019;21(6):e13300.

31. Ryan C. Computer and Internet Use in the United States: 2016. American Community Survey Reports, ACS-39. U.S. Census Bureau, Washington, DC, 2017.

32. Atske S, Perrin A. Home broadband adoption, computer ownership vary by race, ethnicity in the U.S. Pew Research Center https://www. pewresearch.org/fact-tank/2021/07/16/home-broadband-adoptioncomputer-ownership-vary-by-race-ethnicity-in-the-u-s/. Accessed 2021.

33. Shaw J, Brewer LC, Veinot T. Recommendations for health equity and virtual care arising from the COVID-19 pandemic: narrative review. JMIR Form Res. 2021;5(4):e23233.

34. Ridgway JP, Almirol EA, Schmitt J, Schuble T, Schneider JA. Travel time to clinic but not neighborhood crime rate is associated with retention in care among HIV-positive patients. AIDS Behav. 2018;22(9):3003-8.

Publisher's Note Springer Nature remains neutral with regard to jurisdictional claims in published maps and institutional affiliations. 